\title{
The Ventricular Excitability-Interval Relationship in Early Diastole in Humans: The Influence of the Electrode Configuration During Bipolar Stimulation
}

\author{
LAURI K. TOIVONEN, ALAN H. KADISH, WILLIAM H. KOU, \\ and FRED MORADY \\ From the Divison of Cardiology, Department of Internal Medicine, University of Michigan \\ Medical Center, Ann Arbor, MI
}

TOIVONEN, L.K., ET AL.: The Ventricular Excitability-Interval Relationship in Early Diastole in Humans: The Influence of the Electrode Configuration During Bipolar Stimulation. The purpose of this study was to determine the relationship between ventricular excitability and the extrastimulus $\left(\mathrm{S}_{2}\right)$ coupling interval in early diastole in humans. The influence of the electrode configuration during bipolar stimulation was examined. Ventricular strength-interval curves were constructed using an eight beat drive train at a cycle length of $600 \mathrm{msec}, 2-\mathrm{msec}$ decrements in the $\mathrm{S}_{2}$ coupling interval, and 0.2-mA increments in the current intensity of $S_{2}$ at each coupling interval. A transient dip in the excitation threshold was observed in early diastole during bipolar pacing when the distal electrode was the anode in six of 17 patients (35\%). In contrast, this type of dip was not observed when the distal electrode was the cathode. The excitation threshold at the trough of the dip ranged from 1.0 to $2.2 \mathrm{~mA}$. Unipolar strength-interval curves indicated that a dip occurred with anodal but not cathodal pacing. We postulated that the dip might interfere with the accurate determination of the ventricular effective refractory period by resulting in transient loss of ventricular capture during decremental scanning of diastole with $\mathrm{S}_{2}$. A gap in ventricular capture was produced in all six patients who demonstrated a dip in the bipolar strength-interval curve by selecting an $\mathrm{S}_{2}$ current intensity that fell within the dip. In conclusion, the bipolar strength-interval curve may display an early diastolic dip in the excitation threshold, depending on the electrode configuration. During determination of the ventricular effective refractory period, if the stimulus intensity falls within the range of current intensities encompassed by the dip, a transient early diastolic gap in ventricular capture will be observed as diastole is scanned with $\mathrm{S}_{2}$. If this type of gap is not recognized, the ventricular effective refractory period may be overestimated. (PACE, Vol. 13, July 1990)

strength-interval curve, ventricular excitability, effective refractory period, anode, cathode

\section{Introduction}

Ventricular refractoriness is measured in the clinical electrophysiology laboratory by determining the longest coupling interval at which a ventricular response cannot be elicited by an extrastimulus $\left(\mathrm{S}_{2}\right)$. Refractoriness generally shortens

Address for reprints: Lauri Toivonen, M.D., Helsinki University Central Hospital, Haartmaninkatu 4, SF-00290 Helsinki, Finland.

Received October 5, 1989; revision February 6, 1990; accepted March 27, 1990. in a curvilinear fashion as the current intensity increases. ${ }^{1-3}$ However, experimental studies have revealed that the excitability of ventricular muscle with bipolar stimulation may be paradoxically enhanced during a short phase on the upward slope of the curve relating the stimulation threshold to the extrastimulus coupling interval $\left(\mathrm{S}_{1} \mathrm{~S}_{2}\right)$ near the absolute refractory period..$^{1,2,4}$ This dip in the ventricular excitation threshold occurs with anodal but not with cathodal stimulation., ${ }^{4,5}$

Previous studies on human ventricular refractoriness have not investigated the presence of 
an early diastolic decline in the excitation threshold during bipolar stimulation. ${ }^{3,6,7}$ Therefore, the present study was undertaken to examine the relationship of the excitation threshold to the extrastimulus coupling interval during bipolar and unipolar stimulation in early diastole.

\section{Methods}

\section{Subjects of the Study}

Thirty-eight patients who underwent a clinically-indicated electrophysiology test were the subjects of this study. Selection criteria consisted of a sinus cycle length $>600 \mathrm{msec}$ and the absence of inducible ventricular and supraventricular tachycardia by a single extrastimulus during programmed ventricular extrastimulation. There were 25 men and 13 women, and their mean age was $54 \pm 16$ years. Seventeen patients had coronary artery disease, five patients had a cardiomyopathy, two patients had valvular heart disease and 14 patients had no identifiable structural heart disease.

\section{Electrophysiology Study Protocol}

The study protocol was approved by the Human Research Committee at the University of Michigan. Electrophysiology studies were performed in the fasting, unsedated state after informed consent was obtained and at least five half-lives after discontinuation of all antiarrhythmic medications. At least two $6 \mathrm{~F}$ quadripolar electrode catheters with a $10-\mathrm{mm}$ interelectrode distance (USCI 006042, USCI, Inc., Billerica, MA, USA) were inserted via a femoral vein. A stable catheter position in the right ventricular apex where the bipolar pacing threshold was $1 \mathrm{~mA}$ or less was obtained under fluoroscopic guidance. The right atrium was paced simultaneously with the ventricles in patients who had atrioventricular dissociation during ventricular pacing, in order to prevent sinus capture beats.

Leads I, III, and $\mathrm{V}_{1}$ and the intracardiac electrograms were recorded at a paper speed of 100 $\mathrm{mm} / \mathrm{sec}$ on a Siemens-Elema Mingograf 7 recorder (Siemens-Elema, Solna, Sweden). Stimulation was performed with a programmable stimulator (Bloom Associates, LTD, Narbeth, PA, USA).

\section{Excitability-Interval Relationship}

The relationship between the stimulation threshold of $S_{2}$ and the $S_{1} S_{2}$ interval (strength-interval curve) was determined during early diastole in 38 patients.

Programmed stimulation was performed using an 8-beat drive train at a cycle length of 600 msec, a 3-second intertrain interval and stimuli 2 msec in duration. The drive train stimuli were maintained at a current strength of twice the late diastolic threshold. The $\mathrm{S}_{1} \mathrm{~S}_{2}$ interval was initially $20 \mathrm{msec}$ longer than the conventional bipolar ventricular effective refractory period and was shortened in 2-msec steps. At each $S_{1} S_{2}$ interval, the current intensity of $S_{2}$ was initially set at a subthreshold value, and then increased in $0.2-\mathrm{mA}$ steps until capture occurred. The $S_{1} S_{2}$ interval was shortened until a current intensity of $8 \mathrm{~mA}$ failed to capture the ventricle at least five consecutive $S_{1} S_{2}$ intervals. The longest $S_{1} S_{2}$ not capturing the ventricles at $8 \mathrm{~mA}$ was considered to be the ventricular absolute refractory period.

\section{Effect of Electrode Configuration on the Strength-Interval Curves}

To determine the extent to which the strength-interval curve is affected by the electrode configuration during bipolar pacing, strength-interval curves were constructed with the distal electrode serving as the cathode in 21 patients and with the distal electrode serving as the anode in 17 patients. Unipolar pacing was performed in nine patients, delivering cathodal and anodal impulses to the distal electrode. In patients in whom unipolar curves were determined, bipolar curves were also determined, with the distal electrode serving as the anode.

A temporary decline in the strength-interval curve, a dip, was defined as a decrease in the excitation threshold by $\leq 0.4 \mathrm{~mA}$ compared to a threshold at any longer $\mathrm{S}_{1} \mathrm{~S}_{2}$ interval.

\section{Identification of a Gap in Ventricular Capture}

In some patients, a dip in the bipolar ventricular strength-interval curve was observed near the absolute refractory period. We postulated that if bipolar pacing was performed at a current intensity that fell between the peak and trough 
levels surrounding the dip, there might be temporary loss of ventricular capture during decremental scanning of an extrastimulus to measure the ventricular effective refractory period. To test this hypothesis, diastole was scanned with an $\mathrm{S}_{2}$ in decrements of $2 \mathrm{msec}$ in patients who had a dip in the strength-interval curve, using a current strength that was halfway between the threshold
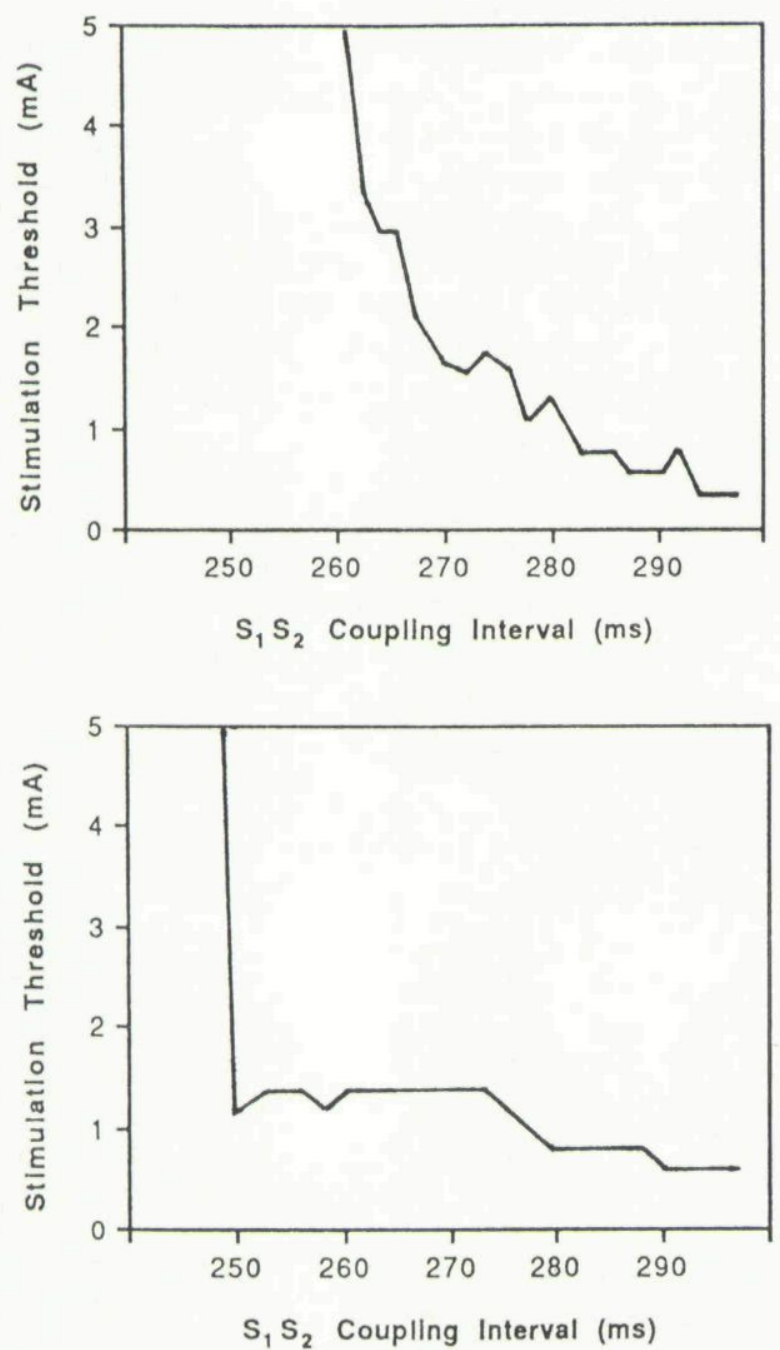

Figure 1. Examples of the ventricular strength-interval curves during bipolar pacing with the cathode located distally. The curves showed a gradual increase in the excitation threshold when the $S_{1} S_{2}$ coupling interval was shortened (upper panel) except in two patients whose curves remained flat until the threshold increased abruptly near the absolute ventricular refractory period (lower panel).

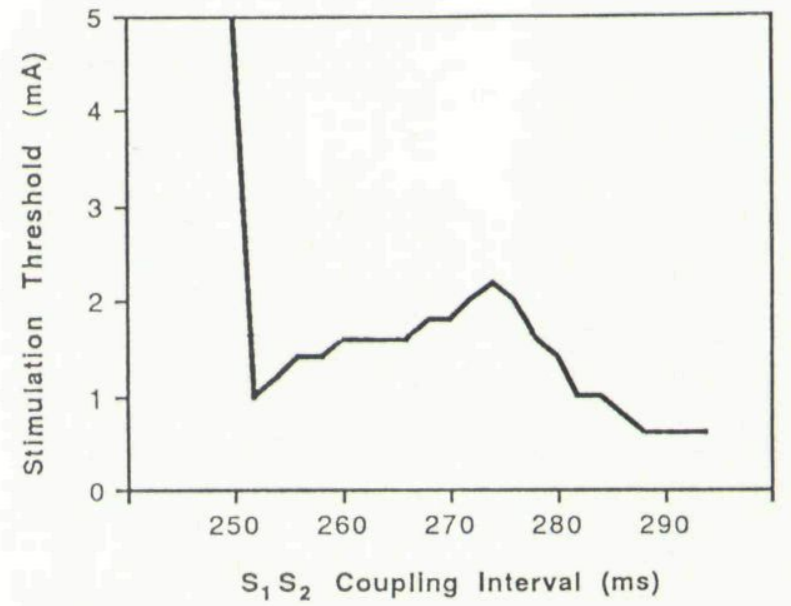

Figure 2. The strength-interval curve during bipolar ventricular pacing with the anode located distally in one of the six patients who demonstrated a dip in the curve. A rise always preceeded the dip.

current preceeding the dip and the threshold current at the trough of the dip. Similar scanning was also performed in patients who did not demonstrate a dip, using a current intensity of twice the late diastolic threshold.

\section{Statistical Analysis}

Student's paired and unpaired $t$-tests were used to examine differences in the thresholds. A P value $<0.05$ was considered significant. Values are expressed as mean \pm standard deviation.

\section{Results}

\section{Bipolar Strength-Interval Curves}

The bipolar strength-interval curves generated with the distal electrode serving as the cathode showed a continuous increase in the excitation threshold when the $S_{1} S_{2}$ interval was shortened in early diastole (Fig. 1).

During bipolar pacing with the distal electrode serving as the anode the ventricular strength-interval curve showed a dip in the excitation threshold before reaching the absolute refractory period in six of 17 patients (35\%, Fig. 2). The peak stimulation threshold at the onset of the dip in the strength-interval curve varied from 2.2 to $5.0 \mathrm{~mA}$. The threshold current at the trough of the dip ranged from 1.0 to $2.2 \mathrm{~mA}$ (Table I). The 


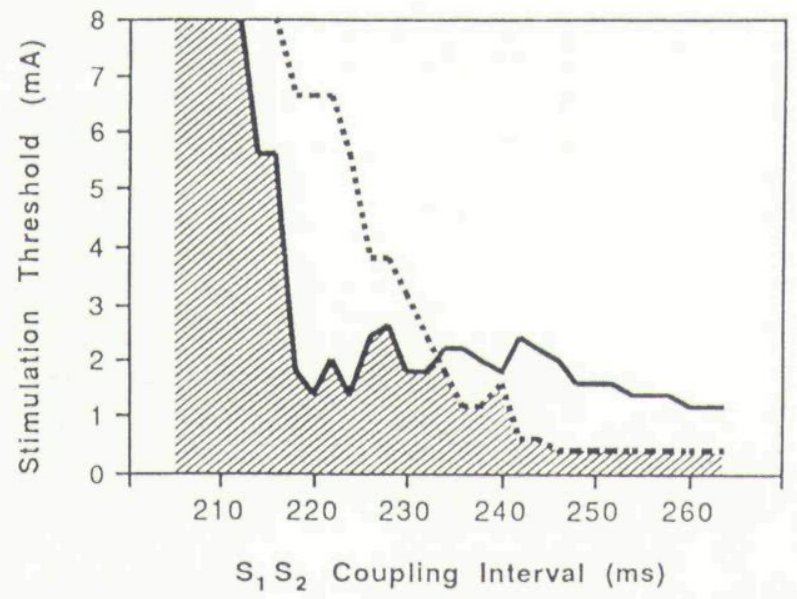

Figure 3. An example of unipolar strength-interval curves. The anodal curve (solid line) demonstrates an early diastolic dip in the excitation threshold, whereas the cathodal curve (dotted line) did not. The strength-interval area where both the cathode and anode were unable to evoke a response is shaded. peak stimulation threshold at the onset of the dip occurred $24 \pm 4 \mathrm{msec}$ before the absolute refractory period, and the trough of the dip occurred 10 $\pm 7 \mathrm{msec}$ before the absolute refractory period. The remaining 11 patients (65\%) displayed no dip in the bipolar strength-interval curve.

\section{Unipolar Strength-Interval Curves}

The anodal strength-interval curves demonstrated a dip in the excitation threshold in eight of nine patients ( $89 \%$, Fig. 3). In contrast, the cathodal strength-interval curves did not demonstrate a dip in any of the patients. The threshold current at the trough of the dip in the anodal strength-interval curves ranged from 1.0 to $4.0 \mathrm{~mA}$ (Table I). In these patients, the bipolar strength-interval curves with the anode distally were compared to unipolar curves. In general, the bipolar strengthinterval curves tracked either with the cathodal or anodal curve.

Table I.

Electrophysiologic Findings in Six Subjects With a Dip in the Bipolar Strength-Interval Curve, and in Nine Subjects in Whom Unipolar Strength-Interval Curves Were Determined

\begin{tabular}{|c|c|c|c|c|c|c|c|}
\hline \multirow[b]{2}{*}{ Subject } & \multicolumn{2}{|c|}{$\begin{array}{c}\text { Lowest } \\
\text { Stimulation } \\
\text { Threshold }^{\star}\end{array}$} & \multicolumn{2}{|c|}{$\begin{array}{l}\text { Stimulation } \\
\text { Threshold at } \\
\text { Onset of Dip }\end{array}$} & \multicolumn{2}{|c|}{$\begin{array}{l}\text { Trough } \\
\text { Stimulation } \\
\text { Threshold }\end{array}$} & \multirow{2}{*}{$\begin{array}{c}\text { Ventricular } \\
\text { ARP ms }\end{array}$} \\
\hline & $\mathbf{m A}$ & $\mathbf{m s}^{\star *}$ & $\mathbf{m A}$ & $\mathbf{m s}^{\star \star}$ & mA & $\mathbf{m s}^{\star \star}$ & \\
\hline \multicolumn{8}{|c|}{ Bipolar Stimulation } \\
\hline 1 & 0.6 & 288 & 2.2 & 274 & 1.0 & 252 & 250 \\
\hline 2 & 0.4 & 274 & 2.2 & 254 & 1.4 & 242 & 234 \\
\hline 3 & 0.4 & 236 & 3.0 & 214 & 1.6 & 202 & 188 \\
\hline 4 & 0.4 & 300 & 2.8 & 280 & 1.6 & 264 & 262 \\
\hline 5 & 0.6 & 260 & 4.0 & 236 & 1.8 & 228 & 206 \\
\hline 6 & 1.2 & 254 & 5.0 & 246 & 2.2 & 224 & 212 \\
\hline \multicolumn{8}{|c|}{ Anodal Stimulation } \\
\hline & 1.0 & 280 & 1.8 & 274 & 1.0 & 266 & 236 \\
\hline & 1.2 & 260 & 2.6 & 228 & 1.4 & 220 & 212 \\
\hline & 0.8 & 306 & 3.0 & 282 & 1.8 & 266 & 264 \\
\hline & 1.0 & 254 & 3.2 & 242 & 2.0 & 230 & 218 \\
\hline & 2.8 & 290 & 4.6 & 272 & 2.6 & 262 & 252 \\
\hline & 1.4 & 260 & 5.2 & 230 & 4.0 & 220 & 218 \\
\hline & 2.3 & 240 & 5.4 & 226 & 4.0 & 216 & 204 \\
\hline & 2.0 & 286 & 8.0 & 256 & 4.6 & 244 & 236 \\
\hline & 1.2 & 272 & - & - & - & - & 236 \\
\hline
\end{tabular}

* Lowest stimulation threshold preceding the dip.

** The extrastimulus coupling interval at which the corresponding stimulation threshold was measured. Abbreviation: ARP = absolute refractory period. 


\section{Demonstration of a Temporary Loss in Ventricular Capture}

Decremental scanning of an extrastimulus at a current strength within the dip in the bipolar strength-interval curve resulted in temporary loss of capture at several intervals $S_{1} S_{2}$ followed by ventricular capture when the $S_{1} S_{2}$ interval was shortened further (Fig. 4). This type of gap in ventricular capture could be demonstrated in each of the six patients in whom the strength-interval curve during bipolar pacing displayed a dip. The duration of the gap in ventricular capture ranged from 4 to $18 \mathrm{msec}$ (mean $12 \pm 5 \mathrm{msec}$, Table II). Ventricular capture resumed again at shorter $\mathrm{S}_{1} \mathrm{~S}_{2}$ intervals extending over a range of 4 to $18 \mathrm{msec}$ (mean $9 \pm 5 \mathrm{msec}$ ). The difference between the apparent and actual effective refractory periods varied from 10 to $34 \mathrm{msec}$ (Table II).

A gap was never observed in the patients who did not display a dip in the bipolar strength-interval curve, with the distal electrode serving either as the anode or cathode.

\section{Thresholds at the Two Electrode Poles}

The late diastolic threshold with bipolar pacing was $0.40 \pm 0.12 \mathrm{~mA}$ when the distal electrode was cathode and $0.59 \pm 0.25 \mathrm{~mA}$ when the distal electrode was anode $(\mathrm{P}<0.01)$. The unipolar late diastolic threshold at the distal electrode was 0.41 $\pm 0.08 \mathrm{~mA}$ with cathodal pacing and $1.64 \pm 0.44$ $\mathrm{mA}$ with anodal pacing $(\mathrm{N}=17 ; \mathrm{P}<0.05)$. The threshold with anodal pacing in the proximal electrode was higher, $3.60 \pm 0.58 \mathrm{~mA}(\mathrm{~N}=21$ : $\mathrm{P}$ $<0.05$ compared to anodal pacing from the distal electrode). The absolute refractory period was shorter during anodal than cathodal stimulation ( $231 \pm 19$ msec vs $240 \pm 18$ msec; $N=9 ; P<0.001)$.

\section{Discussion}

\section{Main Findings}

The results of this study indicate that an early diastolic dip may occur in the curve relating the ventricular bipolar excitation threshold to the
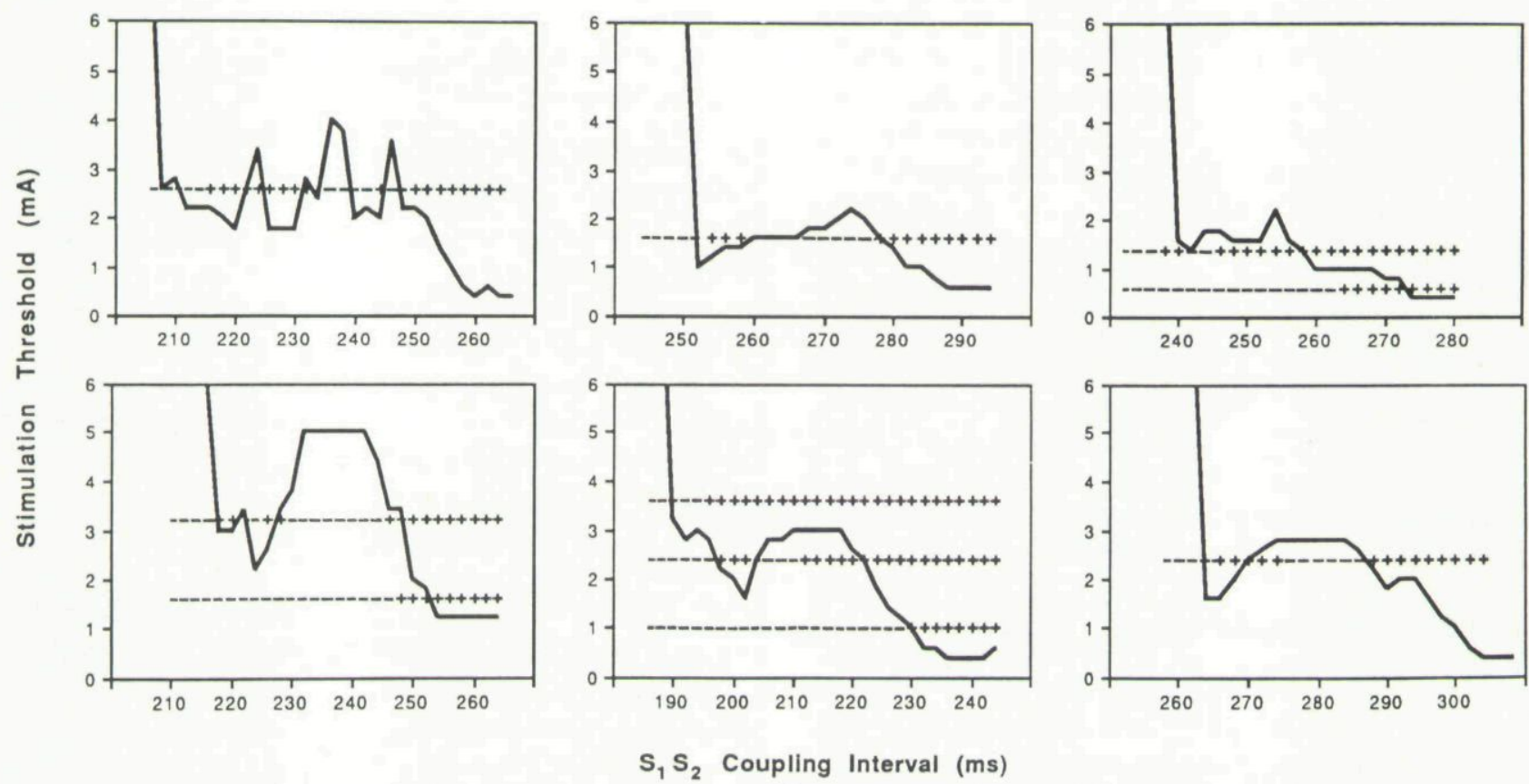

Figure 4. Demonstraton of a gap in ventricular capture during decremental scanning of early diastole with an extrastimulus in the six patients who demonstrated a dip in the strength-interval curve during bipolar pacing with the anode located distally. Capture (+) or noncapture (-) by $\mathrm{S}_{2}$ delivered at a current intensity within the dip is indicated. Temporary loss of ventricular capture coincided with the rise in the threshold preceeding the dip. Capture resumed during the fall in the threshold within the dip, generating a gap in ventricular capture during decremental scanning with $\mathrm{S}_{2}$. Current intensities outside the dip did not produce a gap. 
Table II.

A Gap in Ventricular Capture During Decremental Scanning of an Extrastimulus at a Current Strength Within the Dip of the Bipolar Strength-Interval Curve (Anode Serving as the Distal Electrode)

\begin{tabular}{ccccc}
\hline Subject & $\begin{array}{c}\text { S2 Current } \\
\text { Strength } \\
(\mathbf{m A})\end{array}$ & $\begin{array}{c}\text { Duration of } \\
\text { the Gap } \\
\mathbf{( m s )}\end{array}$ & $\begin{array}{c}\text { Apparent } \\
\text { Ventricular } \\
\text { ERP (ms) }\end{array}$ & $\begin{array}{c}\text { Actual } \\
\text { Ventricular } \\
\text { ERP }(\mathbf{m s})^{\star}\end{array}$ \\
\hline 1 & 1.6 & 18 & 276 & 252 \\
2 & 1.4 & 4 & 244 & 234 \\
3 & 2.4 & 8 & 210 & 196 \\
4 & 2.4 & 12 & 286 & 264 \\
5 & 2.6 & 16 & 248 & 214 \\
6 & 3.2 & 16 & 244 & 216 \\
\hline
\end{tabular}

The subject numbers refer to the same subjects in Table 1.

* The longest extrastimulus coupling interval associated with loss of ventricular capture at the onset of the gap.

** The longest extrastimulus coupling interval associated with loss of ventricular capture after it was resumed at coupling intervals shorter than the gap.

Abbreviation: ERP $=$ effective refractory period.

extrastimulus coupling interval during ventricular pacing in humans. The dip is a result of the anodal contribution during bipolar pacing.

The presence of a dip in the ventricular excitation threshold during bipolar pacing is dependent on the electrode configuration. It was found when the anode was the distal electrode but not when the anode was the proximal electrode.

The early diastolic dip in the strength-interval curve is responsible for a phenomenon that has not been described previously, i.e., a temporary loss of ventricular capture during decremental scanning of early diastole with an extrastimulus to determine the ventricular effective refractory period. Because of a dip in the excitation threshold, ventricular capture may be lost at a particular extrastimulus coupling interval but regained at a shorter coupling interval.

\section{Bipolar and Unipolar Strength-Interval Curves}

Analysis of the strength-interval curves generated by bipolar and unipolar pacing indicates that an early diastolic dip in the bipolar curve may be present if there is a dip in the unipolar anodal curve. The ventricular excitation threshold is lower at the cathode than at the anode, except at the very beginning of diastole. A shorter effective refractory period at the anode permits ventricular capture at intervals where cathodal capture is lost. Previous studies have demon- strated that ventricular excitation actually can occur at the anode and/or cathode during bipolar extrastimulation. ${ }^{4,8}$

\section{Role of the Excitation Thresholds at the Electrode Poles}

Presumably owing to a better contact with the endocardium, the excitation threshold is lower at the distal than a more proximal pole of an electrode catheter. Therefore the chance of ventricular capture increases by positioning the anode distally. Following the common practice of using a stimulation current intensity of twice the late diastolic threshold, the current intensities will generally become lower when the cathode is located distally. This further reduces the likelihood of regaining ventricular capture at the bottom of the dip.

Thus with conventional bipolar pacing, with the cathode located at the distal pole, a dip in the excitation threshold should be rare. However, this phenomenon may occur relatively often if the electrode polarity is reversed and the anode is located at the distal pole. Anodal stimulation results also in ventricular capture with shorter coupling intervals than cathodal stimulation. Prior studies have shown that the ventricle is more vulnerable to fibrillation during anodal extrastimulation due to shorter refractoriness at the anode. ${ }^{9,10}$ 


\section{A Gap in Ventricular Capture During Decremental Scanning of Early Diastole with an Extrastimulus}

The results obtained with decremental scanning of diastole with an extrastimulus at a fixed current strength in patients in whom strength-interval curves previously had been generated demonstrate that an early diastolic dip in the ventricular excitation threshold may be associated with a gap in ventricular capture, depending on the stimulus current strength that is used. A gap in ventricular capture was observed during bipolar pacing in each patient whose bipolar strength-interval curve demonstrated an early diastolic dip and in none of the patients whose bipolar strength-interval curve did not demonstrate this dip. Furthermore, the gap was observed only when the current strength of the extrastimulus was within the range that encompassed the dip in the excitation threshold. Therefore, a gap in ventricular capture is not only specific to patients who demonstrate an early diastolic dip in the bipolar strength-interval curve, but is also specific to current strengths that fall within the dip.

\section{Prior Studies on the Ventricular Excitability- Interval Relationship}

Experimental studies have demonstrated that an early diastolic dip in the ventricular exci-

\section{References}

1. Orias O, Brooks C, Suckling EE, et al. Excitability of the mammalian ventricle throughout the cardiac cycle. Am J Physiol 1950; 163:272-281.

2. Hoffman BF, Gorin EF, Wax FS, et al. Vulnerability to fibrillation and the ventricular-excitability curve. Am J Physiol 1951; 167:88-94.

3. Greenspan AM, Camardo JS, Horowitz LN, et al. Human ventricular refractoriness: Effects of increasing current. Am J Cardiol 1981; 47:244-250.

4. van Dam RT, Durrer D, Strackee J, et al. The excitability cycle of the dog's left ventricle examined by anodal, cathodal, and bipolar stimulation. Circ Res 1956; 4:196-204.

5. Cranefield PF, Hoffman BF, Siebens AA. Anodal excitation of cardiac muscle. Am J Physiol 1957; 190:383-390.

6. Engel RT, Meister SG, Frankl WS. The extent of supernormal ventricular excitability in man. J Electrocardiol 1977; 10:13-17.

7. Mehra R, Furman S. Comparison of cathodal, anodal, and bipolar strength-interval curves with tation threshold is observed consistently during unipolar anodal pacing but not during unipolar cathodal pacing. ${ }^{4.5}$ The pattern of the curves in the present study corresponds to those observed in various animal species. ${ }^{1,2}$ The precise mechanism of the early dip remains unclear, although it has been suggested that the dip in the anodal strength-interval curve is attributable to the hyperpolarizing effects of anodal stimulation. ${ }^{11,12}$

An early diastolic dip in the bipolar strengthinterval curve has not been demonstrated in humans. Only one prior clinical study examined the strength-interval curve that is generated with unipolar anodal and cathodal pacing. As in the present study, an early diastolic dip was present in the unipolar anodal strength-interval curve. ${ }^{7}$

\section{Implications}

The present study indicates that a temporary loss of ventricular capture during bipolar extrastimulation due to an early diastolic dip in the excitation threshold is likely to occur if the distal electrode is used as the anode. The ventricular effective refractory period may be overestimated by approximately $10-30 \mathrm{msec}$ if a temporary loss of ventricular capture is not recognized.

Acknowledgment: We are grateful to Beverly Burgie and Joan Bergeron for their technical assistance.

temporary and permanent pacing electrodes. Br Heart J 1979; 41:468-475.

8. Stevenson WG, Wiener I, Weiss JN. Contribution of the anode to ventricular excitation during bipolar programmed electrical stimulation. Am J Cardiol 1986; 57:582-586.

9. Mehra R, Furman S, Crump JF. Vulnerability of the mildly ischemic ventricle to cathodal, anodal, and bipolar stimulation. Circ Res 1977; 41:159166.

10. Mitamura H, Ohm O-J, Michelsson EL, et al. Importance of the pacing mode in the initiation of ventricular tachyarrhythmia in a canine model of chronic myocardial infarction. J Am Coll Cardiol 1985; 6:99-103.

11. Goto M, Brooks C. Membrane excitability of the frog ventricle examined by long pulses. Am J Physiol 1969; 217:1236-1240.

12. Dekker E. Direct current make and brake thresholds for pacemaker electrodes on the canine ventricle. Circ Res 1970; 27:811-823. 
This document is a scanned copy of a printed document. No warranty is given about the accuracy of the copy. Users should refer to the original published version of the material. 